\title{
Three-space inference from two-space stimulation
}

\author{
JAN B. DEREGOWSKI and DENIS M. PARKER \\ University of Aberdeen, Old Aberdeen, Scotland
}

\begin{abstract}
Oblique contours sloping at $30^{\circ}$ with respect to the horizontal were presented alone, in combination to form chevrons, or with a vertical line to form arrowhead or Y patterns; they were projected onto a screen in the frontal parallel plane and viewed from positions that gave viewing angles of $90^{\circ}$ (normal to the screen's surface), $53^{\circ}$, or $34^{\circ}$. The perceived orientation of the contours, as assessed by a movable arm that the subjects set to be parallel to the obliques, changed monotonically as a function of viewing angle. The change was as great for single obliques as for combinations of obliques within the chevron, arrowhead, and $Y$ patterns. The results of Experiment 1 were extended in Experiment 2, in which obliques at $30^{\circ}$ and $50^{\circ}$ with respect to the horizontal were presented singly or in combination as chevron patterns. It is argued that the results of both experiments indicate that single two-space oblique lines are immediately interpreted as lying in threespace and that the changes in perceived orientation are a consequence of this perceptual inference.
\end{abstract}

The idea that certain two-dimensional (2-D) contour configurations evoke automatic interpretation as 3-D projections is not a new one. It was invoked by Thiery (1896) as an explanation of the effects associated with the MüllerLyer and Ponzo figures. It was implicit in a suggestion by Tausch (1954) that there can be cues present in a 2-D display that trigger a "constancy" correction because of a transfer of learning from 3-D configurations. It was also the basis of Kristof's (1961) and Gregory's (1963; Gregory \& Harris, 1975) explanations that illusory distortion in well-known 2-D figures such as the Müller-Lyer, Poggendorf, and Ponzo were caused by inappropriate automatic triggering of perspective interpretations, which in turn activated "constancy" corrections. The hypothesis that line drawn patterns are particularly prone to perceptual distortion seems intrinsically plausible. Contours on flat surfaces are not particularly significant features of the natural visual environment, and we are unlikely to have evolved any special visual mechanisms for dealing with them. The visual system must process them by means of the mechanisms that are used for processing properties of real objects in real space, yet these skeletal configurations lack the concrete properties of real surfaces that would fix their dimensions.

It is easy to accept that visually rich $2-D$ patterns, photographs, and paintings are interpreted as 3-D structures, since these seem to approximate more closely the richness of the real world's optical array. However, the interpretation of visually rich 2-D images may depend on the extraction of fairly primitive feature relations from within them which may yet be highly predictive of realworld properties. This principle of " non-accidentalness" (Lowe, 1985) or the "likelihood principle"' (Lowe, 1990;

Correspondence should be addressed to J.B. Deregowski, Department of Psychology, King's College, University of Aberdeen, Old Aberdeen, AB9 2UB Scotland.
Rock, 1983) argues that within an image, feature relationships such as collinearity, symmetry, parallelism, and cotermination, which are robust across a reasonable range of viewpoints, are interpreted by the visual system as referring to real-world properties (Barrow \& Tenenbaum, 1981; Biederman, 1987; Lowe, 1985); that is, they are interpreted as being 3-D structures. Even if such feature relationships are "accidentally" arranged, they give rise to the perception of significant structure by the viewer (Barrow \& Tenenbaum, 1981; Kanade, 1981). These results suggest that primitive structural features of the image may be crucial to its 3-D interpretation. Two experiments reported by Deregowski \& Parker (1988) reinforce the view that impoverished visual patterns may be subject to transformations that are similar to those found with visually rich arrays. Thus, when changes in the perceived inclination (heading) of a pattern of three coterminating lines, which represented the major axes of Vermeer's "The Music Lesson," were investigated as a function of angle of view, they were found to be $70 \%$ of the values obtained when a copy of the picture itself was viewed (see Deregowski \& Parker, 1988, for details). This result suggests that the information provided by the contours that indicate the positions of the sidewall and back wall of the picture are the major factors in determining the perceptual transformation that occurs when one views the picture, and further, that the bare contour arrangement, unsupported by texture gradient, familiarity, or size information is the crucial spatial element in triggering the perceptual change. Since the change in perceived inclination of the pictorial structure depends on its interpretation as a projection of three-space structures (Goldstein, 1987; Halloran, 1989), there is a clear suggestion in this result (Derȩgowski \& Parker, 1988 ) that the simple pattern of coterminating lines triggered a three-space interpretation.

Changes in three-space interpretation of 2-D configurations can be seen in other simple patterns. For example, the configuration in Figure $1 \mathrm{~A}$ shows that the perceived 


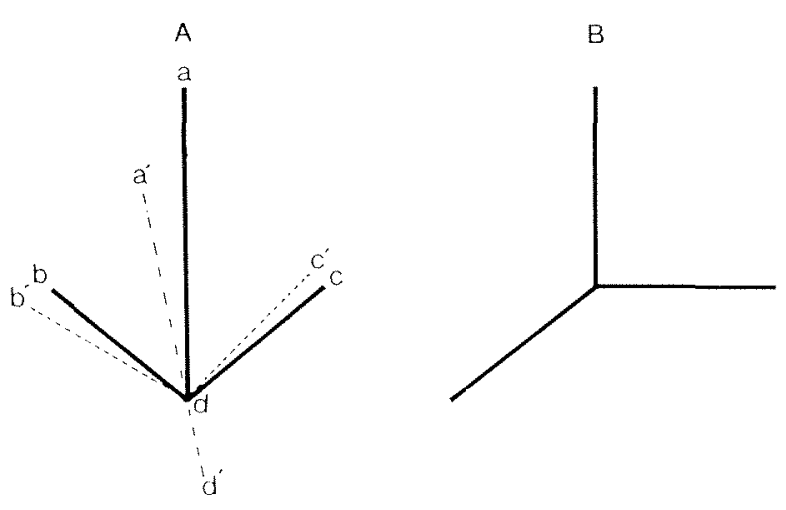

Figure 1. (A) When viewed from the straightahead, the arrowhead configuration appears symmetrical; angles $a d c$ and $a d b$ are equal. If the configuration is seen from a significant angle to one's left, with the surface of the picture remaining in the frontal parallel plane, an apparent change in the relative size of the angles occurs; adc is seen as smaller than $a d b$ (e.g., $a d c^{\prime}$ and $\left.a d b^{\prime}\right)$. The bisector of this apparent angle $a^{\prime} d^{\prime}$ has rotated in the direction indicated. (B) The $Y$ configuration used in the Derȩgowski and Parker (1988) study.

orientations of the two fins of the arrowhead vary significantly with angle of view. When viewed from straight ahead, the left and right acute angles are perceived as equal, but when they are viewed from a significant angle to one's left side, the right-hand angle appears to be more acute than the left. This effect is reversed if the angles are viewed from one's right; the left-hand angle is then seen as more acute than the right. The whole configuration is perceived as if it were a 3-D corner, which, when viewed from straight ahead, has a symmetrical apex (one where the true vertical of the configuration and the line that bisects the obtuse angle of the fins are exactly coincident). When viewed from a fairly steep angle of regard (say, $45^{\circ}$ ), the figure is perceived as having rotated, so that a line drawn to bisect the apparent angle of the fins and continued through their point of cotermination slopes significantly with respect to the vertical, and the corner appears to point toward the observer (see Figure 1A). Thus, although changes in viewing angle result in a changed retinal orientation for the oblique contours, this does not predict the perceptual effect. Increasing the angle of view results in the retinal orientation of all oblique contours moving toward the vertical. Indeed, if the angle of view becomes very steep-less than $10^{\circ}$-this is exactly what happens perceptually, and the fins of the arrowhead are seen to fold in toward the vertical, producing a real effect of anamorphosis. However, over an intermediate range of views, which include those reported in this paper, the oblique that is closer to the perceiver appears to assume an angle that is steeper than that seen from the frontal view, while the oblique that is farther from the perceiver appears to have a shallower angle (an effect contrary to the direction of any slight retinal change). The combined effect is that the corner is perceived to rotate toward the viewer's new position-that is, the heading of the apex of the corner changes.
It is not clear from the information presented above which aspect of the figure is sensitive to the effects of angle view, and, by implication, which aspect can be said to drive the inference from two-space to three-space. It could be the whole configuration, or it could be one or more of its contributory features. If one considers the three coterminating contours used in the Dereggowski and Parker study (1988), it becomes clear that vertical and horizontal contours in three-space do not change their apparent orientation with horizontal changes of the line of sight, as oblique contours do. However, these stable vertical and horizontal contours may form a reference against which the apparent change in the oblique is judged. But it is equally possible that a solitary oblique line appears to change its spatial orientation, and thus its inclination (heading) with respect to the viewer, as if it were a line non-coplanar with the surface of the picture. The experiments reported below were designed to investigate which properties of coterminating configurations determine the apparent spatial transformations with viewpoint.

\section{EXPERIMENT 1}

\section{Method}

Subjects. Eighteen adult subjects ( 9 men and 9 women) drawn from a paid subject panel of inhabitants of a university city were used.

Stimuli and Apparatus. Six different stimuli were used. These were simple geometric figures, back projected onto a screen so that the oblique elements were always $22 \mathrm{~cm}$ long and the vertical element, where present, $60 \mathrm{~cm}$ long. In all the figures, the obliques sloped at $30^{\circ}$ to the horizontal. The stimuli were (1) an oblique sloping left to right; (2) an oblique sloping right to left; (3) two obliques forming a V; (4) two obliques forming an inverted $\mathrm{V}$, (5) two obliques forming a $\mathrm{V}$, the angle of the $\mathrm{V}$ having a vertical bisector; and (6) two obliques forming an inverted $V$, the angle of the $\mathrm{V}$ having a vertical bisector.

The first of the two figures listed above were the simple obliques. The next pair were $\mathrm{V}$ forms constructed from these obliques, and the third pair were the same V's with vertical lines added. In all stimuli, the point of confluence of the lines appeared at about the same position on the projection screen, $125 \mathrm{~cm}$ above the floor; when simple obliques were presented, they occupied the same position as did the corresponding obliques used as elements in more complex figures, incorporating upright V-elements. All stimuli are shown in Figure 2 . Note that the six figures contain a total of 10 oblique lines. (i)

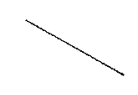

(iv)
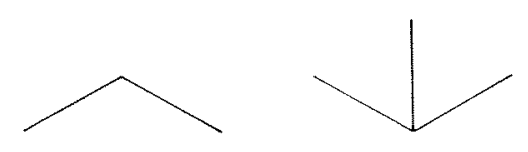

(iii)

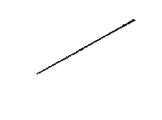

(v)

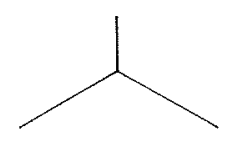

Figure 2. The six patterns used in Experiment 1. 

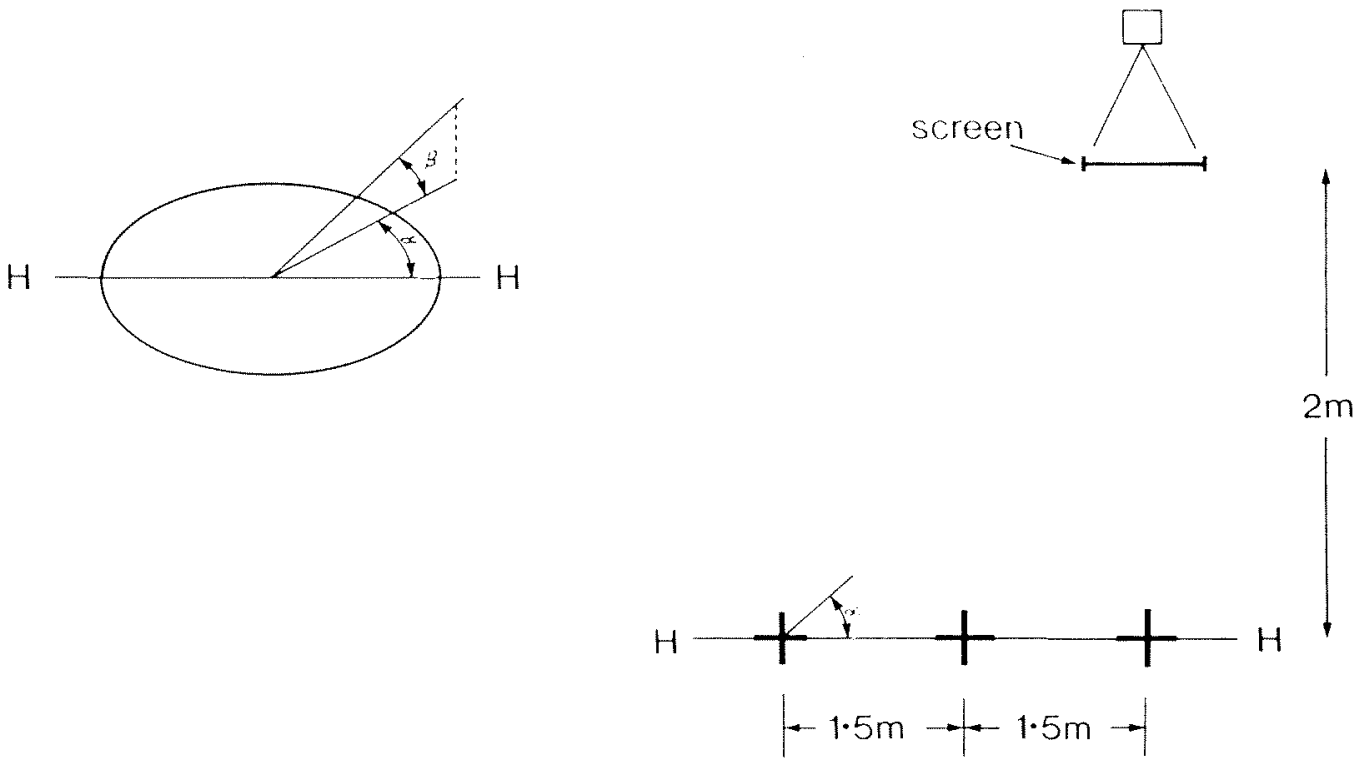

Figure 3. The experimental arrangement used in Experiment 1. The subjects were positioned at one of the three viewing positions on a line $(\mathrm{H})$ parallel to the projection screen and $2 \mathrm{~m}$ from it. Two angles were measured-the setting of the pointer in the horizontal plane (horizontal angle), and the setting of the pointer in the vertical plane (angle of tilt).

A line of traverse was marked on the floor of the laboratory parallel to the plane of the screen, at a distance of $2 \mathrm{~m}$ from it. The three observation stances were marked on this line. One was directly in front of the screen and the other two were at distances of 1.5 and $3 \mathrm{~m}$ to the left, respectively, giving viewing angles of $90^{\circ}, 53^{\circ}$, and $34^{\circ}$. A plan of the layout is shown in Figure 3. Because variations in viewpoint affect the perceived orientation symmetrically (see Deręgowski \& Parker, 1988), viewpoints varying only in one direction from the line normal to the screen were used.

A special measuring apparatus was used. It consisted of a movable pointer hinged at one end, which could be set at any orientation in space. It was placed on the three locations on the traverse indicated in Figure 3. The pointer's fixed end was situated $110 \mathrm{~cm}$ above the floor, and the pointer was connected to a computer so that its angular setting, in both the horizontal and the vertical planes, could be recorded.

Procedure. The subjects stood in a randomly assigned position on the traverse, immediately behind the adjustable arm, and set the pointer so that it appeared to them parallel with the element indicated by the experimenter. No attempt was made to restrict the subjects' head movements; they were merely told that they had to remain in their places behind the apparatus and adjust the pointer so that it was parallel to the contour indicated on the screen. (It is clear from other experiments [cf. Deregowski \& Parker, 1988] that changing the position with respect to the subject does not affect the pattern of results.) The setting of the pointer was recorded in terms of two angles: its angle of tilt to the horizontal plane (angle of tilt, $\beta)$, and the angle between its projection in the horizontal plane and a datum line within the plane parallel to the plane of the screen angle (see Figure 3). Six subjects responded from each of the three stances, and each subject occupied only one stance so that the possibility of carryover effects from other stances could be eliminated.

At each stance, four random series of 10 figures were presented to the subject (so that the perceived orientation of every oblique line in the series of slides could be assessed). A response was obtained to only one of the obliques contained within each figure on any specific presentation, that oblique being indicated by the ex- perimenter. Thus, in the course of a session, four responses were obtained to each oblique.

\section{Results}

The angles of tilt with respect to the horizontal plane (hereafter called angles of tilt) and the angles within the horizontal plane (hereafter called horizontal angles) obtained at each stance in response to the oblique lines in each of the figures were averaged. The averages were used to calculate the perceived angle contained between the obliques forming each pair. It was assumed, on the basis of earlier work (Dereggowski \& Parker, 1988), that this angle is likely to be subject to systematic variation with the observers' position only in the case of the horizontal angle measurements and not in the case of measurements of angles of tilt. In fact, the mean angles of tilt for the three stances at lateral displacements of $0,1.5$, and $3.0 \mathrm{~m}$ were small, being $3.2^{\circ}, 3.8^{\circ}$, and $-0.7^{\circ}$, respectively, and did not in fact vary significantly either with viewing stance $[F(2,15)=1.1, p>.33]$ or with pattern configuration $[F(4,60)=0.7]$. The interaction between these two factors was also nonsignificant $[F(8,60)=1.1, p>.35]$.

For the purpose of the further analysis concerning the apparent rotation of the figures in the horizontal plane, the inclination (heading) of the bisector of the horizontal angle to the plane of the screen was determined (see Figure 1A). Our reasons for using this measure can be explained as follows. Perceived changes affecting the $\mathrm{V}$ figures could have two simple forms:

1. The unaltered figure could appear to rotate in space, in which case the perceived angle between its limbs would be unaffected but the perceived direction of the bisector 
of this angle would change its apparent inclination (heading) with respect to the observer.

2. The figure's shape could appear to change when the perceived angle between its limbs changes (e.g., by foreshortening as the angle of view became steeper), in which case the bisector of the angle would always be seen as occupying the same position but the angle between the limbs would appear to change.

These two kinds of apparent change could, of course, be simultaneous. Measuring both these changes enables one to determine the nature of apparent transformation of these figures. Hence, both the internal angle and the direction of its bisector were calculated.

With these measures, the relationship between responses made to single obliques and those made to $\mathrm{V}$ figures incorporating such obliques can be investigated. If the apparent changes in the perceived angle between two obliques presented separately and the perceived inclination of the bisector of that angle were the same as in the corresponding $\mathrm{V}$ figures, it would be plausible to assume that the perceived changes of the $\mathrm{V}$ figures derive directly from the perceived changes in orientation of the corresponding simple obliques. In these calculations, the responses obtained to the obliques presented on their own (configurations $\mathrm{i}$ and $\mathrm{ii}$ in Figure 2) were paired and treated as if they formed a single cohesive figure. The perceived boundaries of this enclosed angle, and of the inclination of its bisector, indicate changes in two important attributes of the perceived figure-its size and its inclination (heading).

Significant differences were obtained when these two horizontal measures were analyzed. There was no systematic effect of viewing stance on the judged size of the horizontal angle $[F(2,15)=1.2, p>.30]$, but there was a highly significant effect of the type of pattern $[F(4,60)$ $=23.2, p<.001]$. The interaction of the two factors was not significant $[F(8,60)=2.1, p>.05]$. It appears, therefore, that the perceived contained horizontal angles (angle sizes) of the patterns were not affected by the observers' position but were affected by the nature of the pattern. Application of the Tukey test (at 5\%) shows that the configurations fall into two groups. The inverted-V stimuli were perceived as containing a smaller angle than all of the other figures were perceived to contain-including the notional figure created by summing the effects of the two isolated oblique lines. The mean perceived contained angles are shown in Figure 4A. The results indicate that the perceived size of the angle depends on the orientation of the figure; it is significantly less for the inverted- $\mathrm{V}$ forms than for the $\mathrm{V}$ forms. The composite figures, however, are perceived in the same manner as are the $\mathrm{V}$ forms, although there is no prima facie reason for assuming this rather than the inverse percepts.

Analysis shows that the inclination (heading) of the horizontal bisector was affected by the observers' viewing angle $[F(2,15)=51.4, p<.001]$ but not affected either by the type of pattern $[F(4,60)=2.4, p>.05]$ or by interaction of these two variables $[F(8,60)=2.0$, $p>$.10]. The mean values of the angle of inclination of the bisector are $89^{\circ}, 74^{\circ}$, and $59^{\circ}$ (Figure 4B). They decline monotonically with the increase of lateral displacement (more acute angles of view). Thus the data show that the observers, at different stances, perceived the angle as not differing significantly in size but as turning round so that the heading of its bisector "followed" them.

Derȩgowski and Parker's (1988) observations suggest that observed transformations that result from changes in
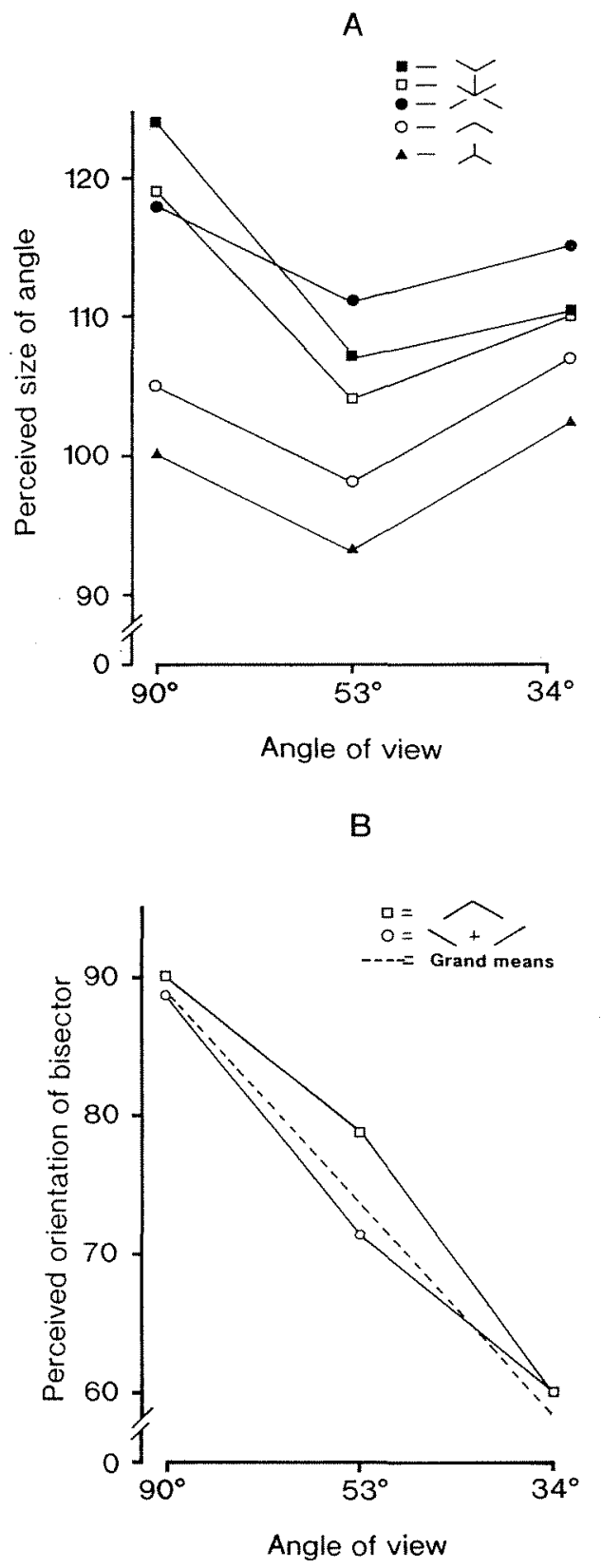

Figure 4. (A) Measured size of the enclosed angle for the patterns shown, as a function of angle of view. (B) Inclination of the apparent bisector of the angle enclosed by the obliques. The dashed line shows the grand means (all figures averaged). All the figures are not plotted separately, because the 5 curves would lie so close together that they would be dificult to distinguish. 


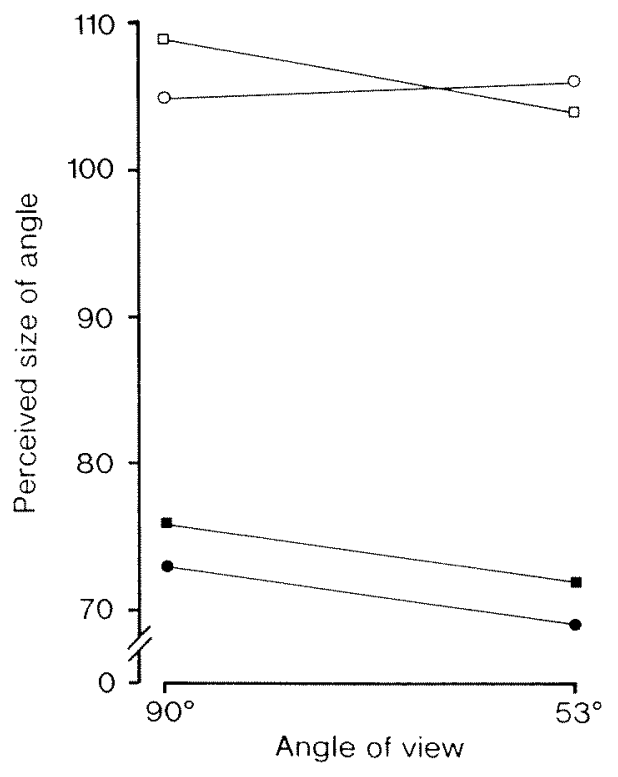

Figure 5. Estimated size of the angle enclosed by the obliques in Experiment 2. Open symbols, $120^{\circ}$ configuration; closed symbols, $80^{\circ}$ configuration. See Figure 6 for other details.

viewing angle are dependent on the orientation at which the stimulus line is drawn. Since in their experiment the horizontal line did not change in apparent orientation, and since other observations indicated that a vertical line also remains perceptually stable, it was necessary to confirm that the results obtained were not unique to the angle at which the lines were slanting. To this end, a second experiment was carried out.

\section{EXPERIMENT 2}

\section{Method}

Subjects. Twelve adult subjects ( 6 men and 6 women) were drawn from a paid subject panel.
Stimuli and Apparatus. Four stimuli were used; the two identical inverted-V stimuli and the two single-line stimuli used in the first experiment (oblique slope $=30^{\circ}$ ), and an analogous set differing only in the orientation of the obliques (slope $=50^{\circ}$ ).

The apparatus from the first experiment was used, but the adjustable arm was placed in only two of the stances used previously, immediately in front of the projector screen and at $1.5 \mathrm{~m}$ lateral displacement, giving viewing angles of $90^{\circ}$ and $53^{\circ}$. Six randomly allocated subjects responded from each of the stances. Each subject responded to two presentations of each stimulus, the stimuli being presented in a random sequence.

\section{Results}

The obtained data were treated exactly as in Experiment 1.

Analysis of the measures of the angle of tilt again shows no systematic effect on the angle size of the subjects' stance $[F(1,10)=1.9, p>.18]$, the angle size $\left(80^{\circ} \mathrm{vs}\right.$. $\left.120^{\circ}\right)[F(1,10)=0.0, p=\mathrm{n} . \mathrm{s}$.$] , or the type of stimulus$ $[\mathrm{F}(1,10)=0.1, p=\mathrm{n} . \mathrm{s}$.$] . The interactions between these$ factors are also not significant.

An analysis of the horizontal angle measures yielded, as anticipated, only one significant factor-angle size $[F(1,10)=252, p<.001)$. The mean estimates of the two angles to the nearest degree were $106^{\circ}$ and $73^{\circ}$ for the objective sizes of $120^{\circ}$ and $80^{\circ}$, respectively (see Figure 5).

The analysis of the bisector measure yielded the anticipated stance effect $[F(1,10)=21.3, p<.001]$ and a significant interaction $[F(1,10)=6.6, p<.03]$ between the figure's angle size $\left(80^{\circ}\right.$ vs. $\left.120^{\circ}\right)$ and viewing stance (Figure 6). The latter unexpected effect necessitated further analysis of the data. This showed that the interaction was due to the consistent differences in the angle of the bisector between the $80^{\circ}$ and $120^{\circ}$ obliques when subjects viewed the configurations from the frontal $\left(90^{\circ}\right)$ stance $[F(1,10)=8.7, p<.02]$, the mean settings being $93^{\circ}$ and $91^{\circ}$, respectively. This difference is small in magnitude and it is difficult to see its perceptual significance.

It is apparent that in Experiment 2, as in Experiment 1, the perceived change in the orientation of the single ob-

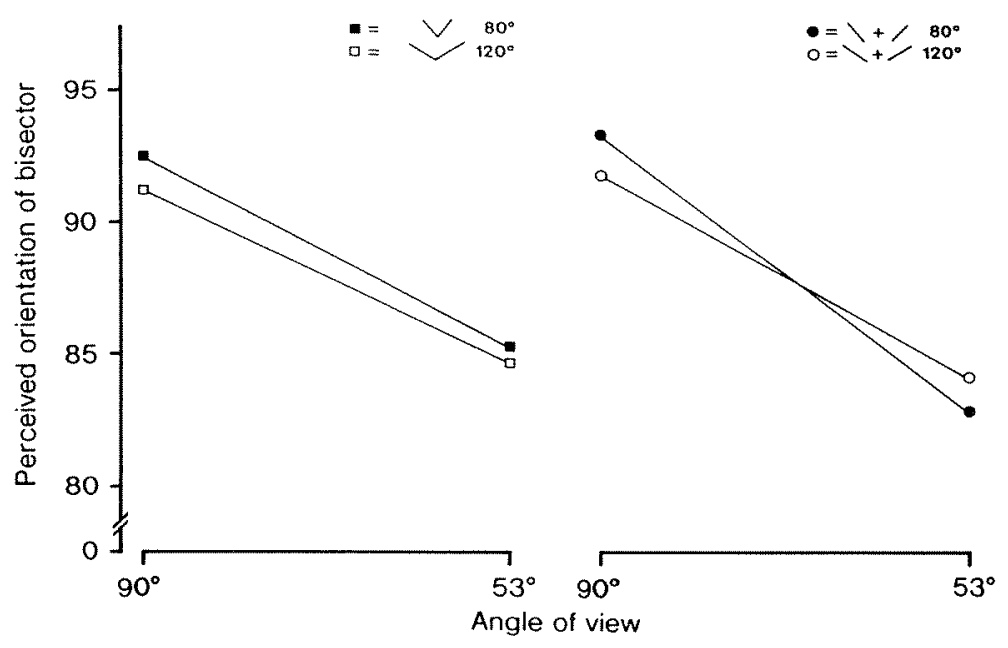

Figure 6. Inclination of the apparent bisector of the angle of the obliques, as a function of viewing position in Experiment 2. 
liques, as assessed by the bisector's inclination, was as great as that for the $\mathrm{V}$ patterns. It is unlikely that responses made to simple obliques were affected by the context of composite figures within which they were presented, because the stimuli were presented in four different random sequences of 10 . Within each set, each oblique was presented once and the requested response had an equal chance of being preceded by a request for a response to either a congruent oblique or its enantiomorph. Furthermore, when, in Experiment 2, two angles of obliquity were used, the results were consistent with those obtained in Experiment 1.

\section{DISCUSSION}

The results of these two experiments indicate that angle of view significantly alters the perceived inclination (heading) of oblique contours. This effect occurs within a context in which the perceived size of the contained angle in the figures does not alter significantly with viewing angle. The latter finding is important, because it suggests that any simple change in retinal projection cannot explain the effect. These experiments also provide support for the view that projections of certain two-space configurations are routinely processed as if they were projections from three-space, since the direction of change in single obliques is consistent with the direction of change in pictorially depicted objects (Deregowski \& Parker, 1988; Halloran, 1989). This conclusion is based on the perceived rotation of the static figures in the transverse plane, which was measured in the present experiments by calculating the inclination (heading) of the bisector of the perceived angle. Viewing angle has a significant effect on the perceived inclination of all the configurations used in these experiments-the patterns of three and two coterminating lines, and the single obliques. The latter finding is particularly interesting, because the perceived rotation of the single obliques appears to determine the rotation of the configurations as a whole; when the contained angle of the two single oblique lines is calculated together with the inclination of its bisector, the degree of rotation is similar to that of the coterminating figures. This suggests that a single oblique line implies a threespace interpretation. Such a conclusion would certainly be consistent with everyday experience, wherein obliques in the frontal parallel plane are infrequent relative to those which arise as a consequence of surfaces and edges receding in depth that project as obliques on the retina. Contour arrangements involving obliques may then be a particularly powerful cue to aid recovery of three-space object properties from two-space configurations.

Under the conditions of the present experiments, the pointer used by the subjects to indicate the perceived orientation of the obliques could be set in any orientation, and the subjects were simply instructed to set it parallel to the indicated line. Yet every subject set the pointer to indicate that the obliques were receding in a plane normal to the screen's surface and at an average elevation of $2.1^{\circ}$ to the horizontal. Thus, in terms of the perceived rotation of the angle of the chevron and other configurations, and in terms of the spontaneous behavior of the subjects in setting the position close to the horizontal, there is evidence that these simple figures are treated as three-space configurations.

The overwhelming consistency of changes in the perceived inclination (heading) of the angle in the horizontal plane with viewing position and the absence of any evidence showing consistent changes of the angles of tilt under the same conditions merits examination. When observers move along a line parallel to a plane displaying an oblique and are permitted to move their heads and eyes freely, the optical information about the orientation of that oblique does not remain constant, because the orientation of its retinal of projection is affected by the subjects' stance on the traverse. In addition, they perceive the relative angle of the screen on which the oblique is displayed and its distance from the observation stance. In consequence of these vectors, the oblique was consistently perceived as changing its inclination in the horizontal plane. This interpretation, although geometrically consistent with changes in the perception of an oblique in a horizontal plane in 3-D space (say, changes in the perception of a rod laid on the floor as a subject walks past), is not the only geometrically acceptable interpretation; an infinity of segments in addition to a horizontal segment can yield a specific location in pictorial space, and the line merely defines a plane on which these segments lie. Consistent choice of the horizontal setting must therefore result from considerations other than purely geometric ones derived from the retinal projection of the oblique. It is possible to argue that the horizontality in itself has a dominant effect on perception, derived from the importance of the horizontal in a phylogenetic context, and that this effect is augmented by the oberver's experience. This assumption was made by Segall, Campbell, and Herskovits (1966) in their extensive cross-cultural study inspired by Brunswick's (1956) theory of probabilistic functionalism. They proposed that the vertical element of the horizontal/vertical illusion figure implies perceptually a receding horizontal, and does so especially forcefully to inhabitants of open plains. This hypothesis did receive a measure of empirical support, but in considering it, one needs to take into account the limitations of cross-cultural studies of illusions, discussed by Derȩgowski (1980, 1989).

The psychophysical evidence presented here also offers some support for models of pattern recognition which directly recover three-space object properties from simple line and edge configurations in a two-space image (Lowe, 1985, 1987; Walters, 1986). The fact that subjects readily interpret simple line configurations as possessing threespace properties argues for their perceptual importance. Further support for the significance of these figures comes from other sources. Warren and Bashford (1977) have argued that perceived length distortions in the region of coterminating line segments, which they assume underlie the Müller-Lyer illusion, are there to compensate for perspective distortion of the component parts of 3-D objects. Walters (1986) has presented evidence that local 
connectedness of line segments can lead to brightness enhancement of these configurations, which can in turn lead to a hierarchy-based discrimination of the most important edges in the input. There is evidence, then, of three kinds of misperception with respect to simple contours, length (Warren \& Bashford, 1977), brightness (Walters, 1986), and, as indicated in this paper, orientation, although distortions of length have received disproportionate attention because of their unmistakable presence in wellknown illusions (see Robinson, 1972).

The main conclusion that can be drawn from the two experiments reported here is that oblique two-space contours are interpreted as implying three-space edges. Changes in the perceived orientation of single obliques with changes in angle of view are as great as changes when the obliques form chevrons or are paired in an arrowhead configuration. We believe that the distortions of space that are evident when one views pictures from an angle (Deręgowski \& Parker, 1988; Halloran, 1989; Jerison, 1967) are probably also largely determined by the oblique contours present in the images. Our reasons for proposing this are based not only on the findings of Deregowski and Parker (1988), where changes in the inclination (heading) of the oblique member of a pattern of three coterminating lines were $70 \%$ of the values obtained with the full picture, but also on the results reported in this paper, where obliques were found to exhibit as great a change when presented alone as when they were members of twoline or three-line configurations. Furthermore, the direction of change with single obliques is the same as that which obtains when one views more complex pictorial elements (Derȩgowski \& Parker, 1988; Halloran, 1989; Parker \& Deregowski, 1990). Given the overlap between viewing-angle phenomena in pictures and in simple contours, the hypothesis that they are closely related effects, with the latter (simple contour effects) underlying the former (pictorial effects), seems reasonable.

One further factor should be noted. Viewing-angle phenomena affect pictures, line patterns, and single contours. They should also be expected to be apparent in video displays and perhaps also in instrument-panel displays. Informal viewing suggests that this is indeed the case, so perhaps the alignment of the viewer with respect to the display may be an important factor when the viewer must make fine-scale discriminations.

\section{REFERENCES}

Barrow, H. G., * Tenenbaum, J. M. (1981). Interpreting line drawings as three-dimensional surfaces. Artificial Intelligence, 17, 205-244.
Biederman, I. (1987). Recognition by components: A theory of human image understanding. Psychological Review, 94, 115-145.

Brunswick, E. (1956). Perception and the representative design of psychological experiments. Berkeley: University of California Press.

DEREgowsKI, J. B. (1980). Illusions, patterns and pictures. London: Academic Press.

DeREgowsKI, J. B. (1989). Real space and represented space: Crosscultural perspectives. Behavioral \& Brain Sciences, 12, 51-119.

Deregowski, J. B., \& PARKer, D. M. (1988). On a changing perspective illusion within Vermeer's "The Music Lesson." Perception, 17, 13-21.

Goldstein, E. B. (1987). Spatial layout, orientation relative to the observer, and perceived projection in pictures viewed at an angle. Joumal of Experimental Psychology: Human Perception \& Performance, 13 256-266.

GrEGORY, R. L. (1963). Distortion of visual space as inappropriate constancy scaling. Nature, 199, 680-687.

Gregory, R. L., HARris, J. P. (1975). Illusion-destruction by appropriate scaling. Perception, 4, 203-220.

Halloran, T. O. (1989). Picture perception is array-specific: Viewing angle versus apparent orientation. Perception \& Psychophysics, 45, 467-482.

JERISON, J. H. (1967). Apparent motion of a vista: An illusion of perspective. American Joumal of Psychology, 80, 448-453.

KANADE, T. (1981). Recovery of three-dimensional shape of an object from a single view. Artificial Intelligence, 17, 409-460.

Karstof, W. (1961). Über die Einordnung geometrisch-optischer Tauschungen in die Gesetzmässigkeiten der visuellen Wahrnehmung. Archiv fü die gesamte Psychologie: Teil I, 113, 1-48.

LowE, D. G. (1985). Perceptual organization and visual recognition. Boston: Kluwer.

Lowe, D. G. (1987). Three-dimensional object recognition from single two-dimensional images. Artificial Intelligence, 31, 355-395.

Lowe, D. G. (1990). Visual recognition as probabilistic inference from spatial relations. In A. Blake \& T. Troscianko (Eds.), AI and the eye (pp. 261-279). New York: Wiley.

Parker, D. M. \& Derégowski, J. B. (1990). Perception and artistic style. Amsterdam: North-Holland.

Robinson, J. O. (1972). The psychology of visual illusion. London: Hutchinson.

Rock, I. (1983). The logic of perception. Cambridge, MA: MIT Press

Segall, M. H., Campaell, D. T., Herskovits, M. J. (1966). The influence of culture on visual perception. Indianapolis: Bobbs-Merrill.

TAuSCH, R. (1954). Optische Tauschungen als artifizielle Effekte der Gestaltungsprozesse von Grossen und Formenkonstanz in der naturlichen Raumwahrnehmung. Psychologische Forschung, 24, 299-348.

THIERY, A. (1896). Über geometrisch-optische Tauschungen. Philosophische Studien, 12, 67-126.

WALTERS, D. K. W. (1986). A computer vision model based on psychophysical experiments. In E. C. Schwab \& H. C. Nusbaum (Eds.), Pattern recognition by humans and machines: Vol. 2. Visual perception (pp. 87-120). New York: Academic Press.

WARREN, R. M., BAshFord, J. A. (1977). Müller-Lyer illusions: Their origin in processes facilitating object recognition. Perception, 6, 615-626.

(Manuscript received May 3, 1991; revision accepted for publication October $30,1991$. 